Les pièces de circonstance contenues dans ce volume sont pour certaines éditées par F. Roudaut. Peut-être les trouvera-t-on moins nécessaires que les Homilies. Elles n'en donnent pas moins un éclairage significatif des positions d'un Tyard diversement impliqué dans les affaires de son temps. La Lettre au Jésuite Charles le saisit ainsi dans son opposition pamphlétaire à un membre de la Compagnie de Jésus qui, pour sa part, n'est jamais attaquée ; le texte latin, très largement satirique, est traduit dans les notes en bas de page. La Généalogie de Hugues Capet le montre fidèle au roi et, rétablissant l'absolue légitimité des descendants d'Hugues Capet, elle souligne la calomnie de toute autre assertion. Une même volonté d'apporter au pouvoir royal un soutien, mais cette fois au moyen d'arguments théologiques, fonde très probablement la traduction des Advis du diacre Agapet.

F. Rouget édite pour sa part une autre pièce de circonstance singulière connue par un unique manuscrit : les Modèles de phrases, ou Discours pour parler, ou escrire. Familier d'Henri III, Tyard semble avoir été requis par le roi au tout début du règne (1574-1575) afin de lui proposer des modèles de phrases éloquentes. L'éditeur replace ce texte dans l'atmosphère savante que favorisent les goûts pour la rhétorique d'un prince volontiers orateur et convaincu des pouvoirs spécifiques de la parole royale. Tyard envisage tous les registres possibles que définit la rhétorique classique et les adapte aux éventuels besoins de son royal destinataire. La seconde partie du manuscrit ici édité propose des « lettres d'amour » qui répondent au climat galant qui entoure la maréchale de Retz; mais ces lettres apparaissent aussi comme un moyen de parfaire la formation royale en proposant au prince des exemples de lettres familières.

Ce volume des auvres complètes propose donc un contenu varié où chacun appréciera librement les qualités multiples de l'écriture tyardienne. On saluera avant tout le savant et beau travail des éditeurs qui nous offrent de l'aborder aisément.

BRUNO PETEY-GIRARD, Université de Paris 12

\title{
Fictions narratives en prose de l'âge baroque. Répertoire analytique. Première partie (1585-1610)
}

Ouvrage publié sous la direction de Frank Greiner

Paris : Honoré Champion, 2007, « Bibliothèque littéraire de la Renaissance », vol. 71, $995 \mathrm{p}$.

Le Répertoire publié sous la direction de Frank Greiner est imposant par son volume (près de 1000 pages dont 200 d'annexes), par l'abondance des notices et par le nombre 
de collaborateurs - une petite quarantaine — ayant participé à son élaboration. Il se présente comme une «première partie » et annonce une suite pour la période postérieure à 1610 . Du fait de la masse des documents mis à la disposition du lecteur, de la grande qualité des notices, du soin apporté à la réalisation matérielle (peu de coquilles) et de l'importance de cette période charnière (1585-1610) pour l'histoire de la littérature, il ne fait nul doute que ce livre trouvera place dans les rayonnages de nos bibliothèques et deviendra bientôt pour le chercheur en littérature un outil de travail important.

Chaque notice comportel'indication du titre exact de l'ouvrage, une description bibliologique minutieuse, un commode résumé du contenu (plus ou moins détaillé : cela va de la simple indication du sommaire à la description par séquences de l'ensemble d'ouvrages parfois fort complexes ; la tâche est d'ailleurs plus délicate qu'il n'y paraît, car fournir le résumé d'ouvrages comme ceux de Béroalde de Verville relève de la gageure), une indication des sources et une courte notice bibliographique quand il y a lieu. Chaque titre est précédé d'une série plus ou moins longue de pictogrammes destinés à préciser la forme et/ou le contenu de l'œuvre. De prime abord, on a un peu de mal à se faire à cette curieuse série de petits signes et à les « traduire » en un contenu intelligible mais, mis à part ce choix éditorial, chaque notice est remarquablement claire.

Il est à noter que les contributeurs ont pris le soin, rare, de fournir la cote des ouvrages dans une grande bibliothèque (le plus souvent la $\mathrm{BnF}$ ou l'Arsenal) et, plus appréciable encore, de préciser la disponibilité du texte sur Gallica (la liste des ouvrages numérisées est donnée en annexe). L’on regrette simplement que n'ait été retenue que la seule bibliothèque numérique de la $\mathrm{BnF}$. Il s'agit bien entendu de la base textuelle la plus importante mais elle peut dans certains cas être utilement complétée. Ainsi le Voyage des Princes fortunez (1610) est-il disponible en mode texte dans les Bibliothèques Virtuelles Humanistes (Epistemon).

Sur le fond, ce Répertoire s’assigne une double visée, explicitée dans la Préface. Il a bien entendu comme but premier de procurer au lecteur le panorama le plus complet possible d'une littérature qui reste, parce qu'elle a le malheur d'être située de part et d'autre d'une frontière séculaire qui n’a évidemment aucune légitimité, relativement moins étudiée que les « vrais $\gg \mathrm{XVI}^{\mathrm{e}}$ et XVII ${ }^{\mathrm{e}}$ siècles. De ce point de vue, l'entreprise est une totale réussite car à feuilleter les pages de ce répertoire, on est tout dépaysé au beau milieu d'une contrée dans laquelle on croyait tout de même avoir souvent voyagé. L'on est frappé de n'avoir lu finalement qu'un si petit nombre des textes mentionnés et décrits. Sans doute tous ne valent pas la peine qu'on s'y arrête longuement mais tout de même ces textes ont plu (les indications d'édition 
sont là pour le montrer) et, à ce seul titre, ils mériteraient d'être au moins pris en compte comme des réalités et des jalons historiques importants dans l'histoire des goûts littéraires.

Mais faire la somme des œuvres du temps, c'est aussi - et c'est là le second objectif que se propose l'ouvrage — faire surgir du neuf en sortant des sentiers battus des anthologies littéraires. Là encore le pari est gagné car page après page, on prend mieux conscience de l'abondance et de la diversité de la matière narrative de cette période et, en même temps, de la vision extrêmement limitée que nous pouvons en avoir. Se vérifie une fois encore l'intuition de la critique de la réception selon laquelle notre passé littéraire n'est qu'une reconstruction a posteriori, un choix parmi d'autres et un panthéon pour notre temps.

Un autre mérite important de ce Répertoire est qu'il permet de découvrir ou de mieux cerner les contours de certaines subdivisions génériques. Car ce n'est visiblement pas la même chose pour les hommes du temps que d'écrire un « Discours » présenté comme « lamentable », « veritable », « tragique », « merveilleux » ou « merveillable $»$. Chaque épithète révèle des choix esthétiques particuliers et ne trouve son véritable sens que par relation avec l'ensemble des autres. La mise en rapport des textes — facilitée par la structure alphabétique du Répertoire — fait apparaître la pertinence et la signification de ces classifications qui ne sont plus les nôtres. Des nuances deviennent alors plus visibles, surtout si l'on a recours en même temps au classement des œuvres par ordre chronologique, fourni en annexe. Il permet de suivre d'un coup d'œil la vie d'un genre, d'en voir les premiers exemples, l'essor puis le déclin. Pour ces différentes raisons, ce répertoire sera très certainement une véritable mine de sujets de recherche.

Reste l'éternelle question de l'« âge baroque ». L'on peut, bien entendu, être sensible — comme la préface le propose de l'être — à son unité mais l'on peut aussi être frappé de la permanence de formes esthétiques de la Renaissance ou même du Moyen Âge (un article de Michelle Szkilnik pointe d'ailleurs à raison la vigueur des textes médiévaux en plein XVII ${ }^{\mathrm{e}}$ siècle). On note en effet partout l'influence des Amadis et l'on doit bien constater que les paradigmes esthétiques anciens — l'allégorie notamment - perdurent visiblement plus longtemps qu'on n'a l'habitude de le dire comme dans ce Lancelot du Lac de 1591, dans cette Monophile de 1597 ou dans cet Ostrassisme d'amour de 1602 .

Il y a en tous cas dans ce livre matière à susciter de nouvelles mises en perspective des œuvres car à procéder ainsi — en prenant tout simplement les œuvres à partir de leurs éditions - on voit bien l'illusion que peut représenter une vision simplement chronologique de l'histoire littéraire. Une fois éditées, les œuvres ne 
disparaissent pas du jour au lendemain du paysage littéraire, et ce qu'on constate, c'est finalement moins l'apparition de tel ou tel texte que le recyclage continu d'une même matière. Ovide, Boccace ou Marguerite de Navarre semblent être des réservoirs sans fin.

Bref, ce Répertoire fait bien plus que de rendre aisément disponible le contenu de certains ouvrages peu accessibles. En remettant sur le métier, de la façon la plus simple qui soit, l'histoire littéraire, il permet au lecteur de se constituer son propre panorama et lui donne tout bonnement envie d'aller lire ces ouvrages oubliés.

STÉPHAN GEONGET, Université François-Rabelais, Tours

\section{Pirates? The Politics of Plunder, 1550-1650}

Edited by Claire Jowitt. Early Modern Literature in History

Basingstoke, Hampshire and New York: Palgrave Macmillan, 2007. Pp. xiii, 244.

This volume, originating in an academic conference, contains ten chapters and an introduction emphasizing the "permeable boundary between legitimate and illegitimate seaborne activities.” This permeability stems from royal authorization of subjects to take reprisals on foreign shipping through "letters of Marque." One result was that diplomatic conventions were depreciated by privateers who, in the face of inadequate state regulation, adopted piratical roles. Claiming wide geographical and interdisciplinary scope, the editor holds that historiographical and literary images of pirates contributed to colonial discourses that justified empire building between 1550 and 1650 . In that period, piracy "intersects with a variety of larger cultural issues: legal, national, colonial, and those to do with race, religion, and gender."

In his chapter, Christopher Harding gives a legal perspective on the "ambivalent view of piratical activity," contrasting it to the "much more definite criminalization of piracy at the turn of the eighteenth century." The earlier period had pragmatic, not moral, reasons for condemning piracy. Hence, Francis Drake and Walter Ralegh were treated as heroes and criminals. Since before 1700 England had limited naval forces, pirates and colonists often co-existed: they mutually benefitted from smuggling and evading commercial rules. While the navy remained small, piracy could be an instrument of state power; but when the state monopolized naval force, piracy had to be eliminated.

John Appleby describes a formative stage in the development of English piracy in the Caribbean and North America: the spread of buccaneers from south-west England to the coast of south-west Ireland. There, out of the reach of English power, 